for his work on the theory of turbulence and other branches of fluid mechanics, for which he was elected a Fellow of the Royal Society in 1957. Dr. Batchelor was born in Melbourne in 1920 and received his first degree from the University of Melbourne in 1940.
Later he went to Cambridge, where he was elected a Fellow of Trinity College in 1947 and awarded an Adams Prize in 1951. He is editor of the Cambridge Monographs on Mechanics and Applied Mathematics and of the Journal of Fluid Mechanics.

\title{
ENZYMES
}

$\mathrm{W}$ AGENINGEN, situated on the Rhine in eastern Holland, contains 22,000 inhabitants, and about seventy institutes for scientific research. About one-half of these institutes form part of the State Agricultural University of Wageningen, while the other half are government or private institutes.

In these pleasant surroundings, a highly successful conference on enzymes was held by the University of Wageningen during April 6-9. The organizing committee was under the chairmanship of Prof. H. J. C. Tenderloo, with Dr. H. Veldkamp as secretary

An introductory lecture was given by E. C. Slater (Amsterdam), who emphasized the central place of enzymology in biochemistry. In his view, it is enzymology which gives biochemistry its own methodology, and its own manner of thinking. (Later in the conference, $\mathbf{H}$. Booij (Leyden), speaking as an 'angry young man', provided the necessary corrective against any over-enthusiastic adoption of this proposition by pointing out that other fields of biochemistry existed.) Although there are many different enzymes, the number of different reaction types catalysed by enzymes is quite small. Life, as we know it, involves comparatively few chemical tricks, which are used over and over again, and the aim of biochemistry is to systematize our knowledge of these tricks. A significant step forward in the systematization of enzymology was the appearance in 1958 of the book "Enzymes", by M. Dixon and E. C. Webb. Studies of the mechanism of enzyme action by kinetic and isotopic methods, and by direct chemical studies in which the enzyme is used as a reactant, have confirmed the suggestion made by Michaelis and Menten in 1913 that the enzyme reacts with at least one of the substrates to give an intermediate enzyme-substrate compound. However, in many cases the intermediate enzyme-substrate compound contains only a part, sometimes a small part, of the substrate. This lecture also dealt with the uses which have been made of isolated enzymes as tools in other investigations, in particular in analytical procedures and in the determ. inations of the structures of compounds isolated from natural materials, such as coenzyme $\mathbf{A}$.

C. de Duve (Louvain) reviewed the recent know. ledge of the localization of enzymes within the cell, which has been gained by fractionation of cell homogenates, particularly by differential centrifugation. He pointed out that although the cytologist would regard the preparation of a tissue homogenate as a brutal disruption of the cell, this homogenate is able to carry out very many reactions, and it is possible to localize different reactions in different cell organellæ which can be isolated from the homogenate. He dealt at length with the theoretical basis of the various methods of separation which have been used, and discussed their advantages and disadvantages. The use of 'marker' enzymes for the main fractions of the rat-liver cell nucleus, mitochondria, microsomes and 'soluble' fraction has led de Duve to the discovery that many hydrolytic enzymes are present in granules a little smaller than mitochondria which he has named lysosomes. More recent work using the technique of density equilibrium in heavy water has suggested that at least two enzymes (uricase and catalase) are present in yet another type of granule. The enzymes within the lysosomes are inactive towards their substrates unless the granules are destroyed mechanically or by suspension in hypotonic solution. The lysosomes also discharge their enzymes into the cell when the latter becomes anaerobic, thus promoting autolysis of the cell.

P. Desnuelle (Marseilles) spoke about the chemical structure of proteins and mechanism of action of proteolytic enzymes. He pointed out that although the chemical structures of insulin and of the polypeptides melanocyte-stimulating hormone and adrenocorticotrophic hormone are now established, the reason for the activity of the whole molecule is not yet known. He discussed what is known about the primary, the secondary and the tertiary structures of proteins, emphasizing that although other structures are theoretically possible, the open-chain is the only type which has been clearly demonstrated. Desnuelle described recent work on the activation of the precursors (the -ogens) of the pancreatic proteolytic enzymes, which has shown the importance of limited proteolysis in this process. Actually, the activation of chymotrypsin and trypsinogen is brought about by the splitting of a single bond. But this splitting is likely to be the first and necessary step for deep changes in the tertiary structure of the precursor, bringing two or three residues at the right position in space for building an 'active centre'.

E. C. Webb (Cambridge) surveyed the properties of the various coenzymes, prosthetic groups and activators of enzymes. He pointed out that metals sometimes act as carriers, sometimes promote the combination of the enzyme with the substrate. The effects of anion activators were discussed in relation to his own recent studies of the activation of arylsulphatase by chloride.

A discussion between Dr. Webb and Prof. Slater on whether a valid distinction could be drawn between a coenzyme and a prosthetic group led to the agreed definition that a compound acts as a coenzyme if during the course of its catalysis of the reaction it reacts first with one enzyme and then with another, and as a prosthetic group if it remains bound to the one enzyme during the course of the reaction. The same substance can in different reactions act as a substrate, a coenzyme or a prosthetic group.

Sir Rudolph Peters (Cambridge) diseussed enzyme inhibitors, illustrating the different types by reference to carbon-fluorine compounds (for example, fluorocitrate), arsenicals and organo-phosphorus compounds. The recent studies at Babraham on the mechanism of the inhibition of aconitase 
by fluorocitrate were presented. In the discussion of arsenical inhibitors, emphasis was placed on the different actions of monosubstituted (for example, lewisite, mapharside) arsenicals, which inhibit pyruvate oxidation, and disubstituted (for example, diphenylchlorarsine) compounds which inhibit isocitric dehydrogenase, as well as on the practical application of 2,3-dimercaptopropanol in reversing the inhibition by arsenicals.

The organo-phosphorus inhibitors were discussed in relation to their action on cholinesterase, and to the reversing effect of pyridine-2-aldoxime methiodide. As an example of antimetabolites, Sir Rudolph discussed the antagonism of the effect of serotonin by 1-benzyl-2,5-dimethyl-serotonin.

F. Dickens (London) discussed the regulation of enzymic reactions within the cell with special reference to carbohydrate metabolism. Regulation can be provided by structural barriers within the cell, but also by enzymic mechanisms which do not require structural separation of reactants. Examples of the latter type are given by : (1) control of rate of mitochondrial respiration by the concentrations of inorganic phosphate and adenosine diphosphate; (2) 'feedback' by inhibition of the enzyme by the product of the reaction, for example, inhibition of hexokinase by glucose-6-phosphate; (3) the existence of different mechanisms for breakdown and synthesis, for example, the equilibrium of the uridine diphosphoglucose pathway of glycogen synthesis is much more favourable to synthesis than is the reversal of the phosphorylytic breakdown. Dickens directed attention to situations where the pentose phosphate pathway is particularly active in relation to glycolysis, for example, in the lactating mammary gland, where its function is probably to provide reduced triphosphopyridine nucleotide for fat synthesis, and in the lens where its function is possibly to provide the same compound to keep glutathione reduced.
H. Chantrenne (Brussels) concluded the conference by a discussion of the present status of the investigation of the mechanism of protein synthesis in genoral, and of enzyme synthesis in particular. He pointed out that the earlier sharp distinction between adaptive and constitutive enzymes has been aban. doned. The recent work of Ingram on abnormal hæmoglobins has shown that even the fine details of the protein structure are controlled by the gene. A mutation of a single gene causes a change of one amino-acid in the polypeptide chain. It is conceivable that the deoxyribonucleic acid of the gene contains the information which determines the sequence of amino-acids in the protein chain. This information must be transferred to the ribonucleic acid in the cytoplasm, where the protein is synthesized. The function of the inducer, the presence of which is necessary for formation of an enzyme, is not known. However, it has been shown that it acts in catalytic amounts, not autocatalytically. This suggests that it acts on an enzyme-synthesizing mechanism which is already present, perhaps in an inhibited form. In the discussion of this paper, Desnuelle pointed out that enzyme activity requires a specific configuration (tertiary structure) of the polypeptide chain. The native form of an enzyme is a unique, unstable structure among many more stable structures. It is, therefore, likely that not only the arrangement of the residues along the chains but also the specific folding of these chains are gene-controlled. This must be taken into account in any consideration of enzyme synthesis.

An unusual feature of this conference which had much to recommend it was that the programme called for only two speakers each day, one in the morning and one in the afternoon. This enabled the speakers to give a long introduction (about $45 \mathrm{~min}$.) for the benefit of non-specialists, before a coffee or tea break, after which the speaker continued for a further $30-45 \mathrm{~min}$. A discussion then followed.

E. C. Slater

\section{ENERGY TRANSFER WITH SPECIAL REFERENCE TO BIOLOGICAL SYSTEMS}

$\mathrm{T}$ THE mechanisms by which energy may be transferred from one atom or molecule to another have become a prominent theme of chemical kinetics, photochemistry and radiation physics-as well as of many biological processes. The same fundamental processes and many of the outstanding problems are common to each of these sciences, and those of biological systems, in particular, depend for their solution on development in other fields. The efficiency and generality of energy transfer between molecules has been fully appreciated only quite recently, and the discussion on this subject, which was held by the Faraday Society at the University of Nottingham during April 14-16, was one of those timely meetings for which the Society is noted. It was attended by two hundred physicists, chemists and biologists, about one-quarter of whom were from overseas.

The discussion was divided into two parts, the first concerned with energy transfer processes in general and the second with their occurrence in bio- logical systems. After the president, Dr. E. W. R. Steacie, had welcomed the overseas visitors, the Spiers Memorial Lecture on "Transfer Mechanisms of Electronic Excitation" was delivered by Prof. Th. Förster, of the Technischen Hochschule, Stuttgart. This provided an excellent introduction to the first part of the discussion, which was concerned almost entirely with this topic. The transfer of electronic excitation energy from one molecule to another can be a very efficient process which may occur, not only at every encounter, but even over distances of $50 \mathrm{~A}$. or more. Apart from the 'trivial' process involving emission and re-absorption of light, this transfer can proceed by a radiationless mechanism known as resonance transfer, the interaction between the molecules being one of dipole-dipole coupling and not overlap of electron orbitals which is significant only at much smaller distances. The process is often also referred to as the Förster mechanism, and Prof. Förster gave a clear account of the quantitative theory which he, principally, has developed. He 